\title{
ERRATA
}

\section{Mycobacterium shimoidei sp. nov., nom. rev., a Lung Pathogen \\ MICHIO TSUKAMURA}

National Chubu Hospital, Obu, Aichi, Japan 474

Volume 32, no. 1, p. 67, column 1, line 3 of Materials and Methods: "RM," "OBJ," and "POW" should read "14795/78 Setter," "11098/78 Brooks," and "6357/78 Cockrell," respectively.

\section{Rhodococcus luteus nom. nov. and Rhodococcus maris nom. nov.}

\author{
O. A. NESTERENKO, T. M. NOGINA, S. A. KASUMOVA, E. I. KVASNIKOV, AND S. G. BATRAKOV
}

D. K. Zabolotny Institute of Microbiology and Virology of the Academy of Sciences of the Ukrainian SSR, 252143, Kiev, USSR

Volume 32 , no. 1 , p. 1 , column 1 , line 10 : "(42)" should read "(43)."

Page 2, column 1, line 12 of Materials and Methods: "(17)" should read "(18)."

Page 3, Table 2, column 2, line 1: "IVM" should read "IMV."

Page 6, column 2, line 10 from bottom: $M$. luteum should be enclosed by quotation marks.

Page 8, Table 6, columns 6 through 8: Boxheads should read as given below

\begin{tabular}{|c|c|c|}
\hline \multicolumn{2}{|c|}{ Group II } & \multirow{2}{*}{$\begin{array}{c}\text { "B. maris" } \\
\text { AUCNM } \\
\text { B-464 }\end{array}$} \\
\hline $\begin{array}{l}\text { R. maris } \\
(6 \\
\text { strains })\end{array}$ & $\begin{array}{l}R . \text { maris } \\
\text { IMV } 195\end{array}$ & \\
\hline
\end{tabular}

Page 8, Table 6, column 1, line 16: " $\alpha$-Aminobutyrate" should read " $\gamma$-Aminobutyrate." Page 9, Table 6, column 1 boxhead: "13803" should read "13808."

Page 10, column 1, line 14: "The" should read "These."

Page 11, column 2, reference 4: Should read as given below.

4. Aristovskaya, T. V., M. M. Gollerbach, G. A. Katanskaya. 1962. Microorganisms and their role in formation of soil and increase in its fertility, p. 349-443. In G. L. Seliber (ed.), Great practicum on microbiology. Higher School, Moscow.

Pages 12 and 13, Table 10, column 9 boxhead: " $R$. rhodenii" should read " $R$. rhodnii." 